\title{
SPI-1 encoded genes of Salmonella Typhimurium influence differential polarization of porcine alveolar macrophages in vitro
}

Kamila Kyrova, Hana Stepanova, Ivan Rychlik, Martin Faldyna and Jiri Volf*

\begin{abstract}
Background: Within the last decade, macrophages have been shown to be capable of differentiating toward a classically activated phenotype (M1) with a high antimicrobial potential or an alternatively activated phenotype (M2). Some pathogens are capable of interfering with differentiation in order to down-regulate the anti-microbial activity and enhance their survival in the host.
\end{abstract}

Results: To test this ability in Salmonella enterica serovar Typhimurium, we infected porcine alveolar macrophages with wild-type Salmonella Typhimurium and its isogenic mutants devoid of two major pathogenicity islands, SPI-1 and SPI-2. The induction of genes linked with $\mathrm{M} 1$ or $\mathrm{M} 2$ polarization was determined by quantification of gene expression by RT-qPCR. The $\Delta \mathrm{SPI}-1$ mutant induced a high, dose-dependent $\mathrm{M} 1$ response but a low $\mathrm{M} 2$ response in infected macrophages. On the other hand, wild-type Salmonella Typhimurium induced a low M1 response but a high, dose-dependent M2 response in infected macrophages. The response to $\Delta \mathrm{SPI}-2$ mutant infection was virtually the same as the wild-type strain.

Conclusions: We therefore propose that Salmonella Typhimurium DT104 studied here can polarize macrophages towards the less bactericidal M2 phenotype and that this polarization is dependent on the type III secretion system encoded by SPI-1.

\section{Background}

Salmonella enterica serovar Typhimurium (Salmonella Typhimurium) is a facultative, intracellular pathogen capable of causing severe gastroenteritis in mammals including humans. Although the virulence of Salmonella Typhimurium is a multifactorial phenotype, there are two key virulence determinants specific for S. enterica. To successfully interact with the immune system of the host, $S$. enterica is equipped with two type three secretion systems (T3SS-1 and T3SS-2) encoded by Salmonella pathogenicity island 1 (SPI-1) and Salmonella pathogenicity island 2 (SPI-2), respectively. These secretion systems evolved to deliver S. enterica proteins directly into the cytoplasm of the host cells. T3SS-1 translocates $S$. enterica proteins across the cytoplasmic membrane and promotes $S$. enterica invasion into nonphagocytic cells [1,2]. T3SS-2 translocates Salmonella proteins into the cytoplasm of the host cell across the

* Correspondence: volf@vri.cz

Veterinary Research Institute, Hudcova 70, 621 00, Brno, Czech Republic phagosome membrane and is essential for Salmonella survival and replication inside professional phagocytic cells [3].

Macrophages represent the key cells of host defense against Salmonella as well as other infections. Within the last decade, macrophages have been shown to have a plastic phenotype, which is dependent on microenvironmental stimuli. Based on gene expression, the macrophages can be divided into two major subtypes; pro- and anti-inflammatory. The pro-inflammatory macrophages (also called M1 polarized or classically activated) comprise of inflammatory cells developed under stimulation by LPS and IFN $\gamma$. Such macrophages exhibit high phagocytic and antimicrobial activity and high expression of pro-inflammatory cytokines (e.g. TNF $\alpha$, IL-8, IL-12, IL-23, IL-1) [4]. In contrast, the alternatively activated macrophages (also called M2 polarized) represent a phenotypically less homogenous group with a low phagocytic and bacterial killing ability, low expression of pro-inflammatory cytokines and high expression of non- 
phagocytic receptors. M2 polarized macrophages play an important role in the reparative phase of inflammation but they are also found in connection with parasitic and chronic bacterial infections. Several gene expression studies have shown that macrophages commonly follow M1 polarization in response to infection with a broad spectrum of Gram-negative and Gram-positive bacteria $[5,6]$. However, some bacterial pathogens are able to manipulate macrophage gene expression and induce the M2 program in order to escape the hostile environment present in M1 polarized macrophages [6-8].

In our previous study, we showed that the absence of the SPI-1 T3SS system led to a significantly higher proinflammatory response of porcine alveolar macrophages (PAM) not only at mRNA but also at protein level [9]. This indicated that SPI-1 encoded T3SS might be involved in the suppression of M1 macrophage polarization. However, we did not address to what extent the number of intracellular bacteria influences such signaling and we also did not determine the expression of genes characteristic of M2 macrophage polarization. In the present study, we therefore focused on the expression profile of the antiinflammatory response genes in macrophages in response to Salmonella Typhimurium infection and the relationship of macrophage expression to the number of intracellular bacteria.

\section{Methods}

\section{Bacterial strains and growth conditions}

Salmonella Typhimurium 16E5 belongs to phage-type DT104 and its isogenic $\triangle$ SPI-1 and $\triangle$ SPI-2 mutants were used in the study. Salmonella Typhimurium $\Delta$ SPI1 and $\triangle$ SPI- 2 mutants were constructed using the onestep lambda red recombination of PCR products [10] removing the whole pathogenicity island 1 or 2 . The deletion of SPI-1 comprises removing 36 genes from avrA up to invH, all of which are known or predicted to be related to T3SS-1. The deletion of SPI- 2 comprises removing 44 genes including orf48, orf32, orf245, orf408, ttrACB and the whole T3SS-2 up to the ssaU gene [11]. Before macrophage infection, overnight cultures were diluted $500 \times$ in LB broth and incubated for $6 \mathrm{~h}$ at $37{ }^{\circ} \mathrm{C}$ to reach late logarithmic stage culture with maximally expressed SPI-1 genes [12]. Just before infection the bacteria were washed and re-suspended in sterile $\mathrm{PBS}$ to $\mathrm{OD}=0.3$. Heat inactivated bacteria of wild-type and $\triangle$ SPI-1 mutant were prepared by heating the culture to $65^{\circ} \mathrm{C}$ for $30 \mathrm{~min}$.

\section{Isolation and cultivation of PAMs}

PAMs were obtained from the lungs of clinically healthy pigs immediately after slaughter by sterile bronchoalveolar lavage as described previously [9]. Isolated PAMs (approx. $2 \times 10^{5}$ of PAMs per well of 24-well microplate) were allowed to attach for $2 \mathrm{~h}$ in DMEM (Gibco, USA) supplemented with antibiotics (penicillin $100 \mathrm{U} / \mathrm{ml}$; streptomycin $100 \mu \mathrm{g} / \mathrm{ml}$, and gentamicin $4 \mu \mathrm{g} / \mathrm{ml})$. Two hours later, porcine serum (Invitrogen, USA) was added to a final concentration of $10 \%$. After 16-hours of cultivation, just prior to the infection with Salmonella, the non-adherent cells were washed away and the medium was replaced with DMEM supplemented with $10 \%$ porcine serum and free of any antibiotics. The porcine serum was inactivated $\left(56{ }^{\circ} \mathrm{C}, 30 \mathrm{~min}\right.$ ) and was free of anti-Salmonella antibodies (tested by Salmotype Pig Screen ELISA, Labor Diagnostik, Germany).

Macrophage cell death was determined by release of LDH using a commercial kit (Promega, CytoTox 96 Non-Radioactive Cytotoxicity Assay) according to the instructions of the manufacturer.

\section{Experimental infection}

To study the effect of PAM infection with Salmonella Typhimurium, multiplicity of infection (MOI) 10 was used. However, when the effect of the number of internalized bacteria was analyzed, MOI 2.5 and MOI 40 were used as well. One hour after the infection, $100 \mu \mathrm{g} /$ $\mathrm{ml}$ of gentamicin was added to kill any extracellular Salmonella Typhimurium. After another hour, the medium with $100 \mu \mathrm{g} / \mathrm{ml}$ of gentamicin was replaced with a fresh one containing a bacteriostatic concentration of gentamicin $(15 \mu \mathrm{g} / \mathrm{ml})$ to prevent extracellular Salmonella Typhimurium replication. Salmonella-PAM interaction was terminated 24 hours after the infection when the lysates of the cells in RLT buffer (RNeasy kit, Qiagen) were harvested. The numbers of intracellular Salmonella in PAMs 4 hours post infection were determined after lysis with $1 \%$ Triton X-100 for $20 \mathrm{~min}$. The suspensions were then serially diluted and plated on LB agar. Heat killed bacteria of wild-type and $\triangle$ SPI-1 strains were used as controls at MOI 10 and 40 and LPS at a concentration of $1 \mu \mathrm{g} / \mathrm{ml}$. Negative controls included an assay performed with PAMs without any contact with Salmonella or LPS.

\section{Cytokine gene expression determined by RT PCR}

To verify the consistency of results with the previous study [9], three pro-inflammatory cytokines (TNF $\alpha$, IL8 and IL-1 $\beta$ ) were measured. To test NFKB involvement in this response, NFKBI $\alpha$ was measured as a part of $\mathrm{NF \kappa B}$ direct regulation because this gene is under tight transcriptional control of NFKB [13]. M2 polarization genes were selected based on previous reports. These included IL-4R [14], mannose receptor and CD163 [ 15-17 ]. Transferrine receptor 1 (TfR1) [ 15], tissue inhibitor of metalloproteinases 1 (TIMP-1) [18], tissue metalloproteinases MMP9 and MMP12 [19] were the other genes that were chosen based on their high 
expression in murine or human M2 polarized macrophages. Bruun et al. [19] also report an increase in expression of arginase-1 in M2 macrophages. Finally, IL-10 was measured as the anti-inflammatory cytokine. Hypoxanthine phosphoribosyl transferase I (HPRT) mRNA was chosen as the most stable house-keeping reference gene based on Genorm analysis [20]. The other tested genes which showed less stabile transcription were: beta2-microglobulin, glyceraldehyde-3phosphate dehydrogenase; histone H3; hydroxymethyl-bilane synthase; succinate dehydrogenase complex, subunit A; TATA box binding protein and phospholipase A2.

Total RNA was purified using the RNeasy kit (Qiagen, Germany) according to the manufacturer's instructions. The quantity and quality of RNA was checked spectrophotometrically and by agarose gel electrophoresis. Purified RNA was reverse transcribed with M-MLV reverse transcriptase (200 U, Invitrogen) and oligo-dT primers, and the cDNA was used either immediately or was stored at $-20{ }^{\circ} \mathrm{C}$ until use. Primers IL-1 $\beta$, TNF $\alpha$ and HPRT were adopted from [21] and IL-8 primers were designed according to [22]. The remaining primers were designed using Primer3 software [23] and all the primers are listed in Table 1. RT-PCR was performed with QuantiTect SYBR Green PCR Kit (Qiagen) using the LightCycler 480 (Roche). Expression levels of target genes were determined as follows. The threshold cycle values $\left(C_{t}\right)$ of gene of interest were first normalized to the $C_{t}$ value of HPRT reference mRNA $\left(\Delta C_{t}\right)$ and the normalized mRNA levels were calculated as $2^{\left(-\Delta C_{t}\right)}$.

\section{Statistical analysis}

Data are presented as the mean and standard deviation of results measured from macrophages originating from 8 to 16 pigs. However, as the experiments were performed with independent batches of PAMs originating from outbred pigs, we commonly observed high and low responders. To normalize the data among different macrophage batches, the transcription rates of individual genes were therefore divided by the arithmetical mean of each individual experiment consisting of macrophages infected with wild type Salmonella, SPI1 mutant each at MOI $=10$, LPS and non-stimulated cells. The normal distribution of data was tested and subsequently, oneway ANOVA followed by Tukey's post-hoc test (Prism, Graph Pad Software, La Jolla, USA) were used to compare the differences in transcriptional levels. Significant differences were defined as those with $p<0.05$.

\section{Results}

\section{Invasion and cytotoxicity}

To investigate how the presence of active SPI-1 and SPI2 T3SS affects the early gene expression of porcine macrophages in vitro, PAMs were infected with wild-
Table 1 List of primers used for RT PCR quantification of gene expression

\begin{tabular}{|c|c|c|}
\hline & Sequence 5'-3' & Reference \\
\hline CD163-For & CTTGGGGCAGCGTTGGCAGGAATAG & this study \\
\hline CD163-Rev & ATGCAGGGCTGATGTCCCCTCTGTC & this study \\
\hline HPRT-For & GAGCTACTGTAATGACCAGTCAACG & [21] \\
\hline HPRT-Rev & CCAGTGTCAATTATATCTTCAACAATCAA & [21] \\
\hline IL10-For & TGAAGAGTGCCTTIAGCAAGCTC & this study \\
\hline IL10-Rev & CTCATCTTCATCGTCATGTAGGC & this study \\
\hline IL1 $\beta$-For & GGGACTTGAAGAGAGAAGTGG & [9] \\
\hline IL1 $\beta-R e v$ & CTTCCCTTGATCCCTAAGGT & [9] \\
\hline IL4R-For & TTCAACACTGAAAACCACACCAC & this study \\
\hline IL4R-Rev & GTGTCCACAATGACAATGCTCTC & this study \\
\hline IL8-For & TTCTGCAGCTCTCTGTGAGGC & [22] \\
\hline IL8-Rev & GGTGGAAAGGTGTGGAATGC & [22] \\
\hline MannR-For & GACCAAAAATTGTTGATGCTGA & this study \\
\hline MannR-Rev & GCACCCGTTAGAATCAGGAG & this study \\
\hline MMP12-For & AGAGGAGGCACATCATGGAC & this study \\
\hline MMP12-Rev & CTTCTGGTGACACGATGGAA & this study \\
\hline MMP9-For & CCTTGAACACACACGACATCTTC & this study \\
\hline MMP9-Rev & CCACATAGTCCACCTGATTCACC & this study \\
\hline NFkBla-For & ACGAGCAGATGGTGAAGGAG & this study \\
\hline NFkBla-Rev & TCATGGATGATGGCCAAGT & this study \\
\hline TFRC-For & AATTCAGAGTTGACATAAGGGAGATG & this study \\
\hline TFRC-Rev & AGACGTAGCACGGAAGAAGTCTC & this study \\
\hline TIMP1-For & CAGGAGTTTCTCATAGCTGGACAAC & this study \\
\hline TIMP1-Rev & GTTCCAGGGAGCCACAAAACT & this study \\
\hline TNF $\beta$-For & CCCCCAGAAGGAAGAGTTC & [21] \\
\hline TNF $\beta-R e v$ & CGGGCTTATCTGAGGTTTGA & [9] \\
\hline Arginase1-For & CCAGTCCATGGAGGTCTGTC & this study \\
\hline Arginase1-Rev & GTGTCTTCCCCAGAGATGGA & this study \\
\hline
\end{tabular}

type Salmonella Typhimurium and the isogenic $\Delta$ SPI-1 or $\Delta$ SPI-2 mutants. The number of intracellular bacteria was dependent on the presence of SPI-1 T3SS as the counts of the $\triangle$ SPI- 1 mutant were 2.7 fold lower than the counts of wild-type strain. Counts of the intracellular $\triangle$ SPI-2 mutant did not statistically differ from wild-type Salmonella Typhimurium. When different MOI were used, the numbers of intracellular bacteria increased with increasing MOI although intracellular counts of the $\triangle$ SPI-1 mutant were always lower than the counts of wild-type Salmonella Typhimurium (Figure 1A).

The level of cytotoxicity of bacteria 24 hours post infection was measured by the activity of LDH released from the dead cells into the culture media. Macrophage cytotoxicity of the wild-type Salmonella Typhimurium did not differ from that of the $\Delta$ SPI-2 mutant but both these strains were more cytotoxic when compared to 
A

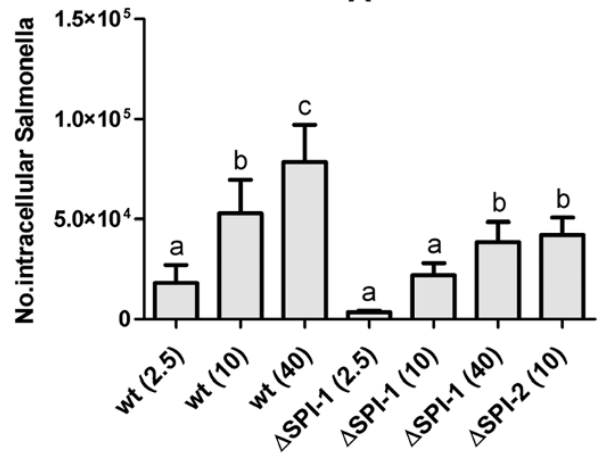

B

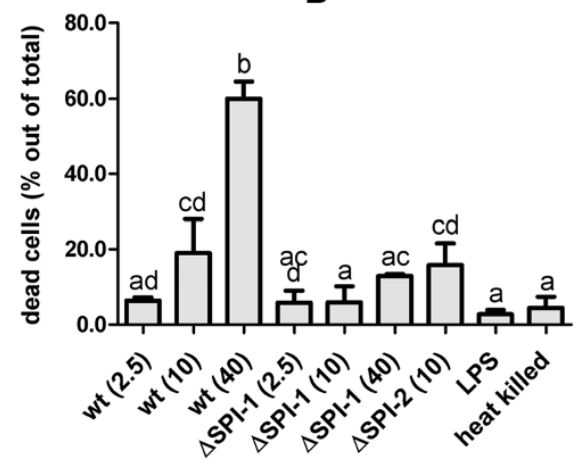

Figure 1 Invasion and cytotoxicity of Salmonella Typhimurium for porcine alveolar macrophages. Panel $\mathbf{A}$, invasion of the wild-type Salmonella Typhimurium (at specified $\mathrm{MOI}$ ) and isogenic $\Delta \mathrm{SPI}-1$ and $\Delta \mathrm{SPI}-2$ mutants into porcine alveolar macrophages $4 \mathrm{~h}$ post-infection. Panel B, cytotoxicity of Salmonella Typhimurium and its isogenic mutants for porcine alveolar macrophages $24 \mathrm{~h}$ post-infection. The data are presented as mean and SD of percentage of $L D H$ released following particular stimulation relative to $L D H$ released from PAMs lysed by freeze-thaw disruption (100\%). Significantly different groups $(P<0.05)$ are marked by different letters ("a" differs from "bc", but not "ab", etc.). Data are presented as mean and SD.

the macrophages infected with the $\Delta$ SPI-1 mutant. In addition, the cytotoxicity of wild-type Salmonella Typhimurium increased with increasing MOI while the $\triangle$ SPI-1 strain did not (Figure 1B).

\section{M1-related signaling at $\mathrm{MOI} 10$}

Similar transcriptional patterns were observed for IL-1 $\beta$, IL-8, TNF $\alpha$ and NFkBI $\alpha$, i.e. the PAMs responded by a higher expression when infected with the $\Delta$ SPI-1 mutant than with the wild-type strain or the $\Delta$ SPI- 2 mutant. All these differences disappeared when the PAMs were stimulated with heat killed bacteria of both wild-type Salmonella Typhimurium or the $\Delta$ SPI-1 mutant.

Except for NFkBI $\alpha$, heat-killed bacteria stimulated a higher response in PAMs than those induced by wildtype Salmonella Typhimurium and the $\Delta$ SPI-2 mutant but lower than those induced by the $\Delta$ SPI-1 mutant. Purified LPS stimulated pro-inflammatory signaling of PAMs to the same extent as the wild-type strain or the $\triangle$ SPI-2 mutant (Figure 2).

\section{M1-related signaling at variable $\mathrm{MOI}$}

Since the behavior of the wild-type strain and the $\Delta$ SPI2 mutant did not differ, the dose dependent experiments were performed only with the wild-type Salmonella Typhimurium and the $\Delta$ SPI-1 mutant. The transcription of M1-related genes did not correspond to a lower or higher MOI of wild-type Salmonella Typhimurium as no significant differences were observed in signaling of PAMs infected at MOI 2.5, 10 or 40 (Figure 2) despite the fact that the numbers of intracellular Salmonella correspondingly increased. On the other hand, the transcription of the same genes showed a clear dosedependent profile in PAMs infected with the $\triangle \mathrm{SPI}-1$ mutant. Stimulation of PAMs with heat killed wild-type
Salmonella Typhimurium and the $\Delta$ SPI-1 mutant at MOI 40 did not result in the induction of any M1 related genes when compared with experiment with MOI 10 (data not shown).

\section{M2-related signaling at MOI 10}

Except for three genes, most of the genes showed a similar transcriptional pattern. These 3 exceptions included MMP9 which was not induced by any stimulation, IL-10 which transcription did not differ following infection with all three strains used, and arginase- 1 which was slightly higher induced by the wild-type than the $\Delta$ SPI-1 or $\Delta$ SPI-2 mutants. The genes with the characteristic pattern for M2-related response comprised IL-4R, mannose receptor, TfR1, CD163, MMP12 and TIMP1 and in all of them, significantly lower expression was observed after PAM infection with the $\triangle$ SPI-1 mutant when compared to the expression in PAMs infected with wild-type Salmonella Typhimurium or the $\Delta$ SPI-2 mutant (Figure 3).

As in the case of M1-related signaling, the differences between the wild-type strain and the $\Delta$ SPI-2 mutant were mostly insignificant with only a few exceptions (IL-4R, TfR1, TIMP1 and arginase-1). However, even in such cases, the differences were minor when compared to those induced by the $\Delta$ SPI-1 mutant. Unlike the proinflammatory genes, the M2-related genes were not induced in PAMs exposed to heat killed bacteria of wildtype Salmonella Typhimurium and $\triangle$ SPI-1 or LPS.

\section{M2-related signaling at variable MOI}

The PAM anti-inflammatory response to infection with the wild-type Salmonella Typhimurium and the $\Delta$ SPI-1 mutant was the opposite of that observed for the pro-inflammatory genes. The transcription of antiinflammatory genes exhibited a clear dose-dependent 

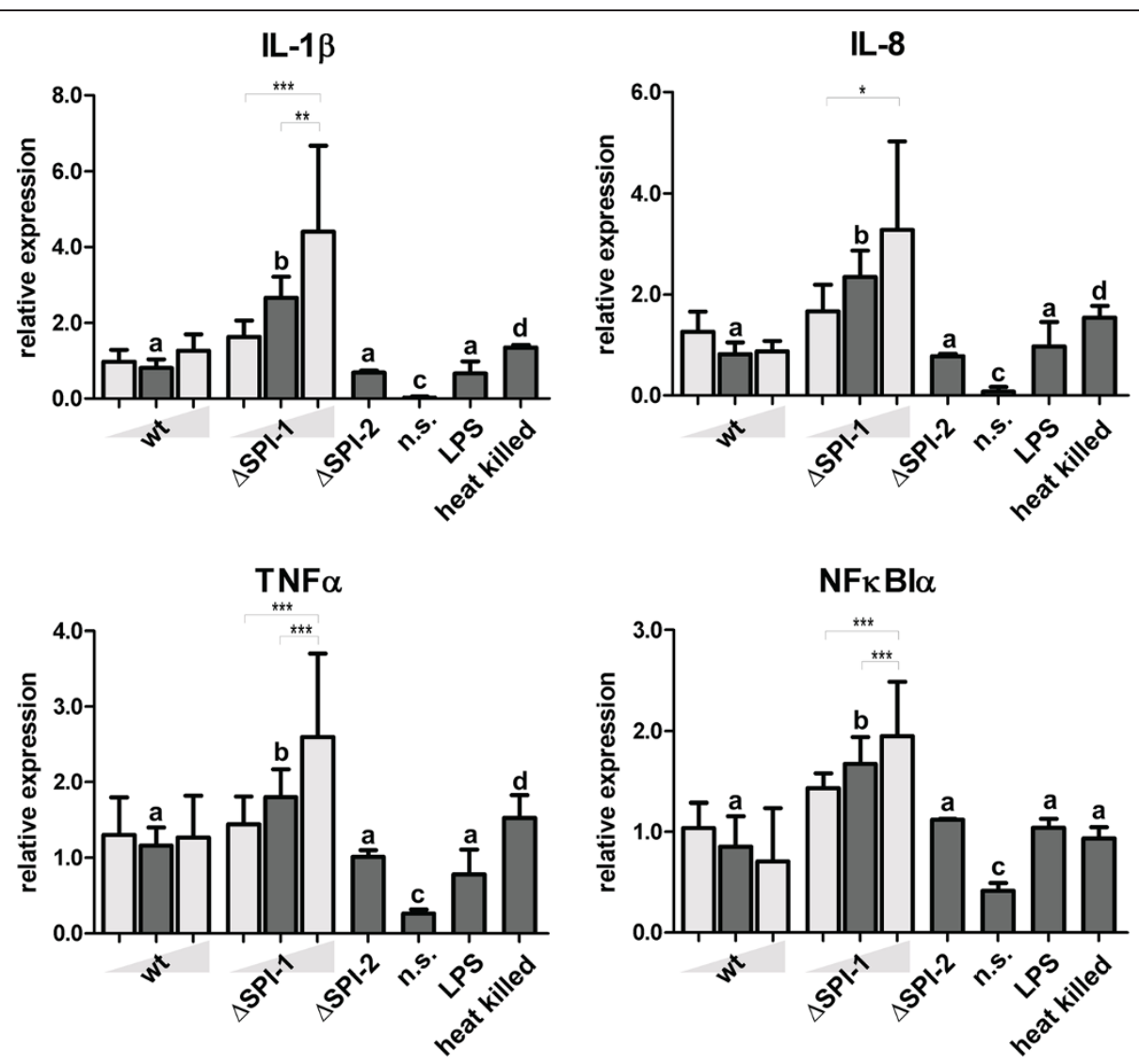

Figure 2 M1-related gene expressions. Dark grey columns represent M1-related gene expression in PAMs 24 hours post infection with the wild-type Salmonella Typhimurium, $\Delta \mathrm{SPI}-1$ and $\Delta \mathrm{SPI}-2$ mutants using $\mathrm{MOI}=10$. Stimulation with LPS from Salmonella Typhimurium (1 $\mu \mathrm{g} / \mathrm{ml})$, heat killed Salmonella Typhimurium and non-stimulated cells (n.s.) were used as controls. Significantly different groups $(P<0.05)$ are marked by different letters ("a" differs from "bc", but not "ab", etc.). Light gray columns represent data for different MOI (=2.5 and 40) of wt strain and $\Delta$ SPI-1 mutant. The statistical significances are marked only among the groups infected with different MOls of the same strain. The significance levels are as follows $0.05\left(^{*}\right), 0.01\left(^{* *}\right)$, and $0.001\left(^{* *}\right)$. Data are presented as mean and SD.

profile after infection with the wild-type strain (Figure 3). On the other hand, the increase in MOI of the $\Delta$ SPI-1 mutant did not induce M2-related genes expression as no significant differences were observed in signaling of the PAMs infected at MOI 2.5, 10 or 40.

\section{Discussion}

In this study, we have shown that the presence of intact SPI-1 genes enables Salmonella polarization of PAMs towards the less bactericidal M2-related response. This conclusion is supported by the fact that the M2 signaling exhibited a dose-dependent response after infection with the wild-type Salmonella Typhimurium whilst M1 signaling was dose independent. In fact, the M1 signaling after infection with the wild-type Salmonella Typhimurium appeared to be a mere response to LPS since the LPS response, response to heat killed Salmonella Typhimurium and responses to the wild-type strain at different MOI were all similar (Figure 2). On the other hand, when SPI-1, but not SPI-2, genes were removed from the Salmonella Typhimurium chromosome, such a mutant was unable to modify macrophage polarization towards the M2. Indeed, infection of PAMs with the $\triangle$ SPI-1 mutant resulted in high, MOI dependent, proinflammatory signaling. Simultaneously, the SPI-1 mutant was essentially unable to induce the expression of M2 related genes, regardless of the MOI used.

Except for MMP9, for which transcription did not react to any kind of stimulation, the transcriptional profiles of the M2 related genes are clustered into two groups. The first group included IL-10 and arginase-1. These two genes were induced by both strains in a dose-dependent manner regardless of the presence of SPI-1. This can be explained by the fact that, although these genes are related to M2 [ 7], IL-10 can be activated by the NF- $\mathrm{kB}$ signaling pathway in the late phases of infection to quell the immune response [24] and arginase-1 is induced following toll like receptor stimulation [25]. These two genes therefore partially share signaling pathways with the proinflammatory genes. The remaining group of mannose 


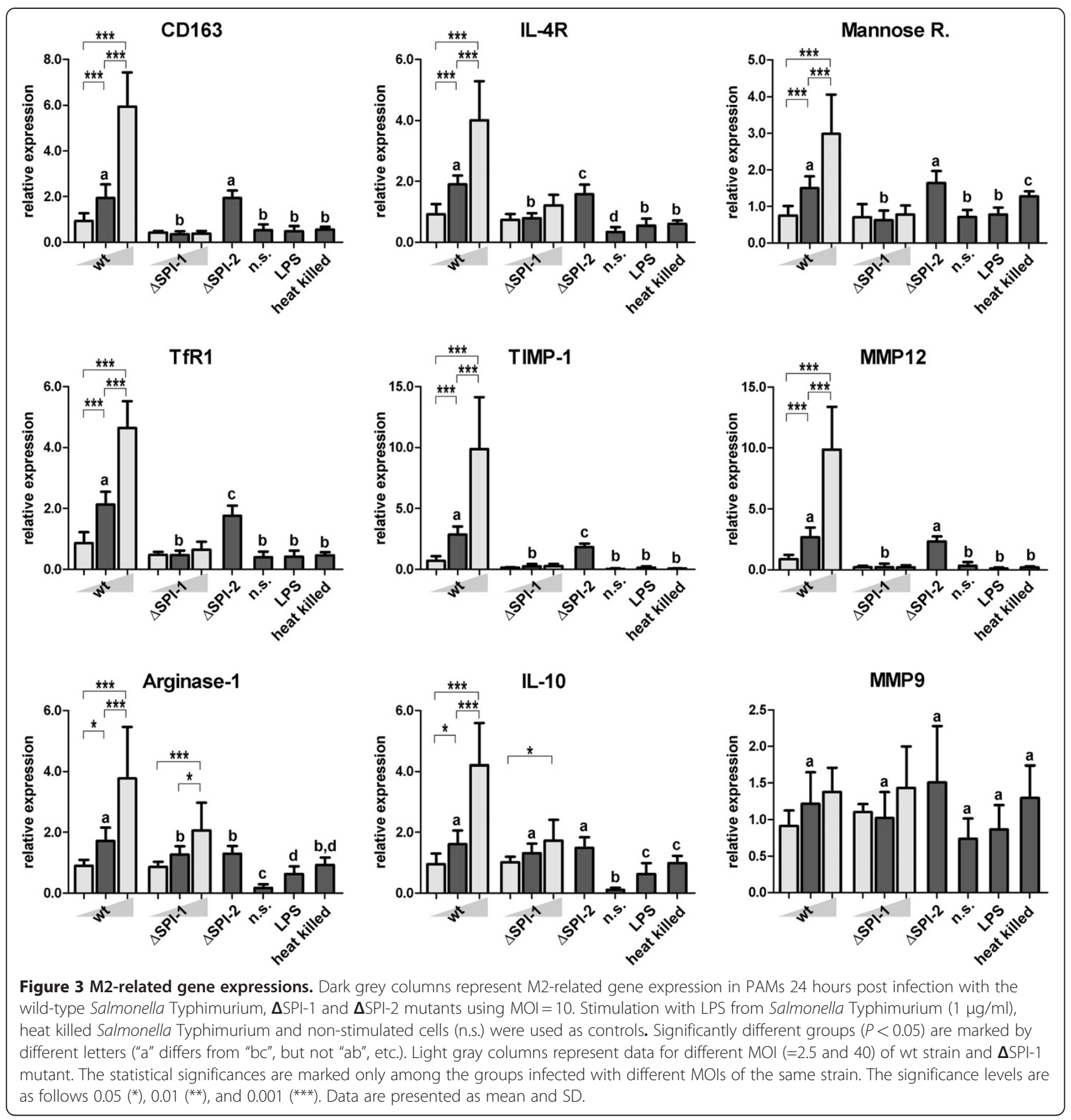

receptor, CD163, TfR1, TIMP-1, IL-4R and MMP12 exhibited a highly similar transcriptional pattern. As this pattern was exactly the opposite of that seen for M1 related genes, we propose these genes belong among the M2 polarization markers in PAMs.

The suppression of pro-inflammatory signaling is macrophage specific. The pro-inflammatory signaling in other cells is dependent on Salmonella invasion, i.e. it is dependent on intact SPI1 [21]. Interestingly, the alternative function of SPI1 genes in relation to differential cytokine signaling may have a clear evolutionary advantage. It has been shown recently that the inflammation induced by Salmonella invading tissue results in host responses which either limit the growth of bacteria different from Salmonella [26] or result in oxidation of intestinal metabolites, which can be used by Salmonella for efficient proliferation at the mucosal surfaces [27]. The invading Salmonella cells thus provide the noninvasive part of the Salmonella population a growth advantage in the gut mucus over the rest of the mucosal 
microbiota. However, once Salmonella crosses the epithelium and comes in contact with macrophages, bactericidal M1 polarization would result in Salmonella eradication and a decrease of pro-inflammatory signaling by the epithelial cells. In addition, M2 polarized macrophages may act against the signaling of epithelial cells and keep an appropriate balance between the epithelial cells and macrophages, which may result in the optimal production of Salmonella required metabolites and limited damage to the host. Interestingly, the ability of Salmonella to polarize the macrophage response towards the M2 might be further potentiated in host adapted serovars. In such cases, this polarization may enable Salmonella to escape macrophage killing and, instead, these serovars can be distributed inside the macrophage across the host's body without being effectively recognized and inactivated.

\section{Conclusions}

All of the presented results support the hypothesis that Salmonella Typhimurium DT104, similar to other bacterial pathogens [6-8], can modify macrophage polarization from the pro-inflammatory and bactericidal M1 rather towards a non-inflammatory M2 and that this polarization is dependent on an intact SPI-1.

\section{Competing interests}

The authors declare that they have no competing interests.

\section{Authors' contributions}

KK contributed to the data collection and analysis, laboratory work, drafting and writing of the manuscript. HS contributed to the design of the study, laboratory work, and drafting of the manuscript. IR and MF contributed to the conception, the acquisition of funds, supervision and drafting of the manuscript. JV contributed to the conception, design, data analysis, laboratory work, drafting and writing of the manuscript. All authors have read and approved the final manuscript.

\section{Acknowledgements}

This work has been supported by the projects MZE0002716202 of the Czech Ministry of Agriculture, AdmireVet project CZ.1.05/2.1.00/01.0006 - ED0006/ 01/01 from the Czech Ministry of Education and project GA524/08/1606 from the Czech Science Foundation. Authors would like to thank Peter Eggenhuizen for his English language corrections.

Received: 28 March 2012 Accepted: 27 June 2012

Published: 20 July 2012

\section{References}

1. Ginocchio CC, Olmsted SB, Wells CL, Galan JE: Contact with epithelial cells induces the formation of surface appendages on Salmonella Typhimurium. Cell 1994, 76:717-724.

2. Zhou D, Galan J: Salmonella entry into host cells: the work in concert of type III secreted effector proteins. Microbes Infect 2001, 3:1293-1298.

3. Freeman JA, Ohl ME, Miller SI: The Salmonella enterica serovar typhimurium translocated effectors SseJ and SifB are targeted to the Salmonella-containing vacuole. Infect Immun 2003, 71:418-427.

4. Verreck FA, De Boer T, Langenberg DM, Hoeve MA, Kramer M, Vaisberg E, Kastelein R, Kolk A, Waal-Malefyt R, Ottenhoff TH: Human IL-23-producing type 1 macrophages promote but IL-10-producing type 2 macrophages subvert immunity to (myco)bacteria. Proc Natl Acad Sci U S A 2004, 101:4560-4565.
5. Boldrick JC, Alizadeh AA, Diehn M, Dudoit S, Liu CL, Belcher CE, Botstein D, Staudt LM, Brown PO, Relman DA: Stereotyped and specific gene expression programs in human innate immune responses to bacteria. Proc Natl Acad Sci U S A 2002, 99:972-977.

6. Nau GJ, Richmond JF, Schlesinger A, Jennings EG, Lander ES, Young RA: Human macrophage activation programs induced by bacterial pathogens. Proc Natl Acad Sci U S A 2002, 99:1503-1508.

7. Benoit M, Desnues B, Mege JL: Macrophage polarization in bacterial infections. J Immunol 2008, 181:3733-3739.

8. Hoffmann R, van Erp K, Trulzsch K, Heesemann J: Transcriptional responses of murine macrophages to infection with Yersinia enterocolitica. Cell Microbiol 2004, 6:377-390.

9. Pavlova B, Volf J, Ondrackova P, Matiasovic J, Stepanova H, Crhanova M, Karasova D, Faldyna M, Rychlik I: SPI-1-encoded type III secretion system of Salmonella enterica is required for the suppression of porcine alveolar macrophage cytokine expression. Vet Res 2011, 42:16

10. Datsenko KA, Wanner BL: One-step inactivation of chromosomal genes in Escherichia coli K-12 using PCR products. Proc Natl Acad Sci U S A 2000, 97:6640-6645.

11. Rychlik I, Karasova D, Sebkova A, Volf J, Sisak F, Havlickova H, Kummer V, Imre A, Szmolka A, Nagy B: Virulence potential of five major pathogenicity islands (SPI-1 to SPI-5) of Salmonella enterica serovar Enteritidis for chickens. BMC Microbiol 2009, 9:268

12. Ibarra JA, Knodler LA, Sturdevant DE, Virtaneva K, Carmody AB, Fischer ER, Porcella SF, Steele-Mortimer O: Induction of Salmonella pathogenicity island 1 under different growth conditions can affect Salmonella-host cell interactions in vitro. Microbiology 2010, 156:1120-1133.

13. Ito CY, Kazantsev AG, Baldwin AS Jr: Three NF-kappa B sites in the I kappa B-alpha promoter are required for induction of gene expression by TNF alpha. Nucleic Acids Res 1994, 22:3787-3792.

14. Herbert DR, Holscher C, Mohrs M, Arendse B, Schwegmann A, Radwanska M, Leeto M, Kirsch R, Hall P, Mossmann H, Claussen B, Forster I, Brombacher F: Alternative macrophage activation is essential for survival during schistosomiasis and downmodulates Thelper 1 responses and immunopathology. Immunity 2004, 20:623-635.

15. Corna G, Campana L, Pignatti E, Castiglioni A, Tagliafico E, Bosurgi L, Campanella A, Brunelli S, Manfredi AA, Apostoli P, Silvestri L, Camaschella C, Rovere-Querini P: Polarization dictates iron handling by inflammatory and alternatively activated macrophages. Haematologica 2010, 95:1814-1822.

16. Goerdt S, Orfanos CE: Other functions, other genes: alternative activation of antigen-presenting cells. Immunity 1999, 10:137-142.

17. Stein M, Keshav S, Harris N, Gordon S: Interleukin 4 potently enhances murine macrophage mannose receptor activity: a marker of alternative immunologic macrophage activation. J Exp Med 1992, 176:287-292.

18. Welch JS, Escoubet-Lozach L, Sykes DB, Liddiard K, Greaves DR, Glass CK: $\mathrm{TH} 2$ cytokines and allergic challenge induce $\mathrm{Ym} 1$ expression in macrophages by a STAT6-dependent mechanism. J Biol Chem 2002, 277:42821-42829.

19. Bruun CS, Leifsson PS, Johansen LK, Jensen HE, Nielsen J, Fredholm M: Expression of matrix Metalloproteinase- 9 and -12 in Porcine lung infections. J Comp Pathol 2012, 146:253-257.

20. Vandesompele J, De Preter K, Pattyn F, Poppe B, Van Roy N, De Paepe A Speleman F: Accurate normalization of real-time quantitative RT-PCR data by geometric averaging of multiple internal control genes. Genome Biol 2002, 3:RESEARCH0034.

21. Volf J, Boyen F, Faldyna M, Pavlova B, Navratilova J, Rychlik I: Cytokine response of porcine cell lines to Salmonella enterica serovar typhimurium and its hilA and ssrA mutants. Zoonoses Public Health 2007, 4:286-293.

22. von der Hardt K, Kandler MA, Fink L, Schoof E, Dotsch J, Brandenstein O, Bohle RM, Rascher W: High frequency oscillatory ventilation suppresses inflammatory response in lung tissue and microdissected alveolar macrophages in surfactant depleted piglets. Pediatr Res 2004, 55:339-346.

23. Rozen S, Skaletsky H: Primer3 on the WWW for general users and for biologist programmers. Methods Mol Biol 2000, 132:365-386.

24. Sarkar S, Han J, Sinsimer KS, Liao B, Foster RL, Brewer G, Pestka S: RNAbinding protein AUF1 regulates lipopolysaccharide-induced IL10 expression by activating IkappaB kinase complex in monocytes. Mol Cell Biol 2011, 31:602-615.

25. El Kasmi KC, Qualls JE, Pesce JT, Smith AM, Thompson RW, Henao-Tamayo M, Basaraba RJ, Konig T, Schleicher U, Koo MS, Kaplan G, Fitzgerald KA 
Tuomanen El, Orme IM, Kanneganti TD, Bogdan C, Wynn TA, Murray PJ: Toll-like receptor-induced arginase 1 in macrophages thwarts effective immunity against intracellular pathogens. Nat Immunol 2008,

9:1399-1406.

26. Santos RL, Raffatellu M, Bevins CL, Adams LG, Tukel C, Tsolis RM, Baumler AJ: Life in the inflamed intestine. Salmonella style. Trends Microbiol. 2009, 17:49Z8-506.

27. Thiennimitr P, Winter SE, Winter MG, Xavier MN, Tolstikov V, Huseby DL, Sterzenbach T, Tsolis RM, Roth JR, Baumler AJ: Intestinal inflammation allows Salmonella to use ethanolamine to compete with the microbiota. Proc Natl Acad Sci U S A 2011, 108:17480-17485.

\section{doi:10.1186/1746-6148-8-115}

Cite this article as: Kyrova et al.: SPI-1 encoded genes of Salmonella Typhimurium influence differential polarization of porcine alveolar macrophages in vitro. BMC Veterinary Research 2012 8:115.

\section{Submit your next manuscript to BioMed Central and take full advantage of:}

- Convenient online submission

- Thorough peer review

- No space constraints or color figure charges

- Immediate publication on acceptance

- Inclusion in PubMed, CAS, Scopus and Google Scholar

- Research which is freely available for redistribution 